\title{
A Soft-computing Assisted Tool to Detect and Analyse Brain Tumor
}

\author{
S. Madhuvanthi ${ }^{1}$, K. Madhumathi ${ }^{2}$, Dr. P. Deepa ${ }^{3}$, Dr. N. Sri Madhava Raja ${ }^{4}$ \\ Department of Electronics and Instrumentation Engineering, \\ St. Joseph's College of Engineering, OMR, Chennai 600119. \\ Tamilnadu, India. \\ ${ }^{1}$ madhuvanthisridhar.163@gmail.com \\ ${ }^{2}$ Mathi7667@gmail.com \\ 33eepachrist@gmail.com \\ ${ }^{4}$ nsrimadhavaraja@stjosephs.ac.in
}

\begin{abstract}
Brain cancer is one of the deadliest diseases in the human community. Early detection is widely preferred to offer better treatment in order to save the patient. In the literature, considerable procedures are proposed and implemented by the researchers. In the proposed work, heuristic approach assisted automated procedure is proposed to segment and analyze the tumor region from the brain Magnetic Resonance Images (MRI). Combination of the Bat Algorithm and Kapur's Entropy is chosen as the initial processing section to improve the visibility of tumor region and the Active Contour (AC) is then chosen as the final processing section to extract the tumor of brain MRI image. Simulation test was executed on the gray scaled brain images obtained from the literature. The reputation of the proposed approach is confirmed against the ground truths supplied by the expert members. The eminence of the proposed procedure is confirmed by computing the image likeliness measures and the area measures. From the results of the experimental work, it can be noted that, the proposed approach offers better result on the gray scaled brain MRI image. Finally, the clinical significance by contributing superior values of image similarity and statistical measure values.
\end{abstract}

Keyword-Brain MRI, Tumor, Entropy, Active contour, Analysis

\section{INTRODUCTION}

In recent years, computed assisted approaches are widely adopted to automate the industrial and medical industry [1-4]. The computer assisted procedures are considered due to its accuracy, simplicity and adaptability. In medical field, these approaches are normally considered to assist the doctors in disease examination, treatment planning and surgery. In medical field, imaging technique is widely considered to analyse the internal body parts and its infections. Cancer is one of the life threatening disease widely affects the internal body organs. Such as brain, bone marrow, lungs, etc.

The main aim of this paper is to propose an automated tool to examine the tumor sections from the brain Magnetic Resonance Image (MRI) database. The image processing literature confirms that, heuristic algorithm approach is efficient in analysing the traditional and medical images [5-10]. Previous works also confirms that, a number of image processing techniques are proposed by the researchers to investigate the cancer with the help of clinical images [11].

In the proposed work, a most successful heuristic approach, known as the Bat Algorithm (BA) is considered to analyse the brain MRI dataset. The heuristic approach based image processing is widely discussed due to its simplicity and lenience in implementation [12-16]. Kapur's entropy is one of the procedures, widely used because of its dominance and elasticity. Kapur's entropy based multi- thresholding was largely discussed by the researchers to extract the important information from the RGB / gray scale test images [17-21].

This paper proposes a computer assisted technique to segment and analyze tumor from the brain MRI dataset. Initially, bi-level thresholding based on the Kapur's entropy is implemented to improve the cancerous division of the brain image. Kapur's approach was initially proposed in 1985 [17]. The combination of Kapur's function and various heuristic algorithms has been presented over the past years for image thresholding applications [20,21].

In this work, recently developed heuristic procedure known as the BA [16] assisted work is employed to enhance the tumor of brain MRI. Later, the Active Contour (AC) [22, 23] based segmentation is implemented to extract the suspicious/enhanced region of brain MRI.

The capability of proposed segmentation task is then confirmed by means of a comparative assessment among the segmented tumor region and the ground truth image offered by the expert member. The experimental 
results verify that, proposed approach is efficient in obtaining the better image quality [24-28] and similarity index [29-31] values. Finally, the proposed approach is validated using the real time brain MRI database obtained from the Bharat scans, Chennai [38]. The real time result also confirms the superiority of the proposed approach. Hence the proposed technique is clinically significant and in future, this toll can be used to assist the doctor to analyse the brain tumor images recorded with Magnetic Resonance technique.

\section{METHODS PROPOSED}

This section presents the details of the methodology proposed in this paper to extract and analyse the tumor from the brain MRI.

\section{A. Bat algorithm}

In the literature, a considerable number of heuristic approaches are proposed to assist the multi-level thresholding process.

The Bat Algorithm (BA) is based on the bio-sonar characteristics of microbats. BA was anticipated by modelling the navigating and hunting potential of bats $[12,16]$. A detailed examination on the BA algorithm can be found in $[14,15]$.

The BA was developed based on the following assumptions:

- All the bats use echolocation to sense distance, prey, and background barriers.

- The bats will fly with a velocity $V_{i}$, at position $X_{i}$ with an emitted frequency $f_{\min }$, varying wavelength $\lambda$ and a loudness $A_{o}$. During the search, the bats will automatically adjust the frequency, wavelength and pulse emission rate $r \in[0,1]$ based on the target distance.

- Along with the above said parameters, the loudness also varies from a large $A_{o}$ value to a constant $A_{\min }$ value.

The Traditional BA (TBA) has three mathematical discrete equations, defining the velocity update, the position update, and the frequency vector as given below:

$$
\begin{aligned}
& V_{i}(t+1)=V_{i}(t)+\left(X_{i}(t)-\text { Gbest }_{i}\right. \\
& X_{i}(t+1)=X_{i}(t)+V_{i}(t+1) \\
& F_{i}=F_{\text {min }}+\left(F_{\text {max }}-F_{\text {min }}\right) \beta
\end{aligned}
$$

where $\beta$ is a random integer in the range $[0,1]$.

From eqn.1, it is noted that, the velocity update mainly depends on the frequency vector. During the optimization search, a new solution for each bat is generated based on the following relation:

$$
X_{\text {new }}=X_{\text {old }}+\varepsilon A^{t}
$$

where $\varepsilon$ is a random numeral in the range [-1,1] and $A$ is the loudness of emitted sound by bats during the exploration of search space.

The minimum and maximum values of the loudness variable $A$ is chosen as $A_{0}=10$ and $A_{\min }=1$ (which decay in steps of 0.01). Other related mathematical representations for loudness adjustment are presented below:

$$
\begin{aligned}
& A_{i}(t+1)=\alpha A_{i}(t) \\
& r_{i}(t+1)=r_{i}(0)[1-\exp (-x)]
\end{aligned}
$$

where $\alpha$ and $\gamma$ are constants typically assigned with a numeral value of 0.75 . 


\section{B. Kapur's entropy}

In image processing literature, variety of scheme is existing to perform the multi-thresholding process [32, 33]. In this paper, analysis is presented for Kapur entropy function based on Bat algorithm. In entropy based approach, the segmentation process finds the optimal threshold, which maximizes the overall entropy.

Kapur's entropy function was originally proposed in 1985 to segment the gray scale image by maximizing the entropy of histogram [17]. In order to get the threshold using Kapur's method; let, $T h=\left[t h_{1}, t h_{2}, \ldots, t h_{\mathrm{k}-1}\right]$ is a vector of the image thresholds.

Then, the Kapur's entropy will be [10, 19 - 21];

$$
J_{\text {max }}=f_{\text {kapur }}(T h)=\sum_{j=1}^{k} H_{j}^{C}
$$

Generally, each entropy is computed independently based on the particular th value.

For multi-level thresholding problem, it can be expressed as;

$$
\begin{gathered}
H_{1}^{C}=\sum_{j=1}^{t h_{h}} \frac{P h_{j}^{C}}{\omega_{0}^{C}} \ln \left(\frac{P h_{j}^{C}}{\omega_{0}^{C}}\right), \\
H_{2}^{C}=\sum_{j=t h_{1}+1}^{t h_{2}} \frac{P h_{j}^{C}}{\omega_{1}^{C}} \ln \left(\frac{P h_{j}^{C}}{\omega_{1}^{C}}\right), \\
\vdots \\
H_{k}^{C} \sum_{j=t h_{k}+1}^{L} \frac{P h_{j}^{C}}{\omega_{k-1}^{C}} \ln \left(\frac{P h_{j}^{C}}{\omega_{K-1}^{C}}\right)
\end{gathered}
$$

where $P h_{j}^{C}$ is the probability distribution of the intensity levels, $C$ is unity (1) for gray level images and $\omega_{0}^{C}, \omega_{1}^{C}, \ldots \omega_{k-1}^{C}$ probability occurrence for $k$ levels. The FA based search arbitrarily adjusts the values of threshold until $J_{\max }$ is reached.

\section{Active Contour Segmentation}

In medical image processing, a class of approaches are available to extract the region of interest from the gray/RGB images $[34,35]$. This subdivision presents the necessary segmentation procedure to extract the cancerous region from the thresholded brain MRI image. In this work, Active Contour Segmentation (ACS) proposed in $[22,23]$ is considered to extract the image. This procedure has three essential steps such as, boundary detection, initial active contour, and final active contour which offer minimized energy. In this work, variable snake model is considered to track similar pixel groups existing in pre-processed image based on energy minimization concept.

The energy function of the snake can be denoted as;

$$
\frac{\min }{C}\left\{E_{G A C}(C)=\int_{0}^{L(C)} g\left(\mid \nabla I_{0} C(s)\right) \mid d s\right\}
$$

where $d s$ is the Euclidean component of length and $L(C)$ is the length of the curve $C$ which satisfies $L(C)=\int_{0}^{L(C)} d s$. The parameter $g$ is an edge indicator, which will disappear based on the object boundary defined as;

$$
g\left(\left|\nabla I_{0}\right|\right)=\frac{1}{1+\beta\left|\nabla I_{0}\right|^{2}}
$$

where $I_{0}$ represents original image and $\beta$ is an arbitrary constant. The energy value rapidly decreases based on the edge value, based on gradient descent criterion ${ }^{12}$.

This procedure is mathematically represented as;

$\partial_{t} C=\left(k g-\left\langle\nabla_{g}, N\right\rangle\right) N$

where $\partial_{t} C=\partial C / \partial t$ represents the deformation in the snake model, $t$ is the iteration time, and $k, N$ are the curvature and normal for the snake ' $C$ '. In this procedure, the snake silhouette is continuously corrected till minimal value of the energy; $E_{G A C}$ is achieved. 


\section{Analysis}

The accuracy of the proposed approach is assessed used two procedures. The thresholding accuracy is assessed using the image quality measures and the segmentation accuracy is assessed using the image area measures.

The well known parameters, such as the Root Mean Squared Error (RMSE), the Peak Signal to Noise Ratio (PSNR) and Structural Similarity Index Matrix (SSIM) are chosen to evaluate the quality of the segmented image.

The mathematical expression is given below:

$$
\begin{aligned}
& \operatorname{RMSE}_{(x, y)}=\sqrt{M S E_{(x, y)}}=\sqrt{\frac{1}{M N} \sum_{i=1}^{H} \sum_{j=1}^{W}[x(i, j)-y(i, j)]^{2}} \\
& \operatorname{PSNR}_{(x, y)}=20 \log _{10}\left(\frac{255}{\sqrt{M S E_{(x, y)}}}\right) ; \mathrm{dB} \\
& \operatorname{SSIM}_{(x, y)}=\frac{\left(2 \mu_{x} \mu_{y}+C_{1}\right)\left(2 \sigma_{x y}+C_{2}\right)}{\left(\mu_{x}{ }^{2}+\mu_{y}{ }^{2}-C_{1}\right)\left(\sigma_{x^{2}}+\sigma_{y^{2}}+C_{2}\right)}
\end{aligned}
$$

where $x$ and $y$ are original and segmented images; $\mu_{x}$ and $\mu_{y}$ are the average values, $\sigma_{x}^{2}$ and $\sigma_{y}^{2}$ are the variance, $\sigma_{x y}$ is the covariance, and $C_{1}=\left(K_{1} L\right)^{2}$ and $C_{2}=\left(K_{2} L\right)^{2}$ stabilize the division with weak denominator, with $L=256$, $K_{1}=0.01$, and $K_{2}=0.03[15]$.

The accuracy of medical image segmentation is commonly confirmed using a relative analysis with the expert segmented image known as the Ground Truth (GT). During this procedure, the image performance metrics, such as Jaccard Index (JI), Dice Index (DI), False Positive (FP) rate and False Negative (FN) rate are computed [28]. These metrics are mathematically expressed as follows;

$$
\begin{aligned}
& \operatorname{JSC}\left(I_{g t}, I_{p m}\right)=I_{g t} \cap I_{p m} / I_{g t} \cup I_{p m} \\
& \operatorname{DSC}\left(I_{g t}, I_{p m}\right)=2\left(I_{g t} \cap I_{p m}\right) /\left|I_{g t}\right| \cup\left|I_{p m}\right| \\
& F P R\left(I_{g t}, I_{p m}\right)=\left(I_{g t} / I_{p m}\right) /\left(I_{g t} \cup I_{p m}\right) \\
& F N R\left(I_{g t}, I_{p m}\right)=\left(I_{p m} / I_{g t}\right) /\left(I_{g t} \cup I_{p m}\right)
\end{aligned}
$$

where, $I_{g t}$ represents the Ground Truth $(\mathrm{GT})$ and $I_{p m}$ stands for the segmented image with proposed method.

\section{RESULTS AND DISCUSSIONS}

This section presents the results obtained with the proposed approach. All the simulations are implemented using the MATLAB software. In this work, three types of the brain MRI datasets are considered for the studies: 1) Image dataset of the Osirix [36], 2) Brain database from the MICCAI [37] and 3) Real time clinical images [38].

Initially the proposed approach is tested on the Osirix dataset presented in Table 1. This image is with the skull region. In this work, three different views of the images, such as the back, mid and side views are considered. Initially, the skull region is stripped using the method discussed in [11]. The bi-level thresholding is executed on the skull stripped image using the BA and Kapur's entropy. Later, the active contour segmentation is applied to extract the tumor section.

The outcome of the proposed approach is clearly shown in Fig 1. Where Fig 1(a) shows the chosen test image, (b) presents the skull stripped image, (c) and (d) depicts the outcome of the thresholding and segmentation approaches respectively and (e) shows the extracted tumor. Similar results are obtained for the other images shown in Table 1.

Table 2 shows the image quality measures obtained with the bi-level thresholded images and Table 3 presents the geometrical measures obtained for the extracted tumor. From these results, it is noted that, proposed approach works well for the Osirix brain MRI dataset. 
Table 1 Test images from Osirix image library

\begin{tabular}{|c|c|c|}
\hline View & Image1 & Image2 \\
\hline $\begin{array}{l}\text { Back } \\
\text { view }\end{array}$ & & \\
\hline $\begin{array}{l}\text { Mid } \\
\text { view }\end{array}$ & & \\
\hline $\begin{array}{l}\text { Side } \\
\text { view }\end{array}$ & & \\
\hline
\end{tabular}

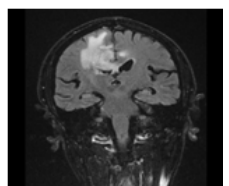

(a) Test image

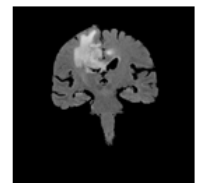

(b) Skull stripped image

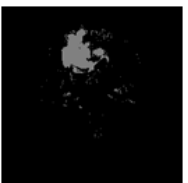

(c) Thresholded image

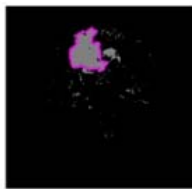

(d) Segmentation

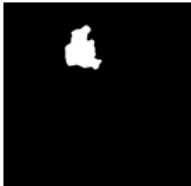

(e) Tumor

Figure 1. Segmentation result obtained with the proposed method

Table 2 Image quality measures obtained during the bi-level thresholding

\begin{tabular}{|c|c|c|c|c|c|c|c|c|c|}
\hline \multicolumn{2}{|c|}{ Image } & MSE & RMSE & PSNR & NCC & AD & SC & MD & NAE \\
\hline $\begin{array}{c}\text { Back } \\
\text { view }\end{array}$ & Image 1 & 1658.6 & 40.7263 & 15.9333 & 0.1691 & 18.7631 & 7.8751 & 111 & 0.8873 \\
\cline { 2 - 10 } & Image 2 & 2071.1 & 45.5090 & 14.9689 & 0.0010 & 21.6953 & 6.1165 & 188 & 0.9001 \\
\hline \multirow{2}{*}{$\begin{array}{c}\text { Mid } \\
\text { view }\end{array}$} & Image 1 & 1829.3 & 42.7701 & 15.5080 & 0.2491 & 16.5475 & 5.0466 & 140 & 0.8184 \\
\cline { 2 - 10 } & Image 2 & 1733.8 & 41.6392 & 15.7408 & 0.3466 & 16.9427 & 3.7103 & 133 & 0.7369 \\
\hline \multirow{2}{*}{$\begin{array}{c}\text { Side } \\
\text { view }\end{array}$} & Image 1 & 1997.6 & 44.6945 & 15.1257 & 0.3002 & 20.4638 & 4.1236 & 129 & 0.7790 \\
\cline { 2 - 10 }$y$ & Image 2 & 2015.5 & 44.8945 & 15.0869 & 0.2959 & 20.5790 & 4.1750 & 130 & 0.7834 \\
\hline
\end{tabular}

Table 3 GLCM measure for the extracted tumor region

\begin{tabular}{|c|c|c|c|c|c|c|}
\hline Image & $\begin{array}{c}\text { Auto } \\
\text { correlation }\end{array}$ & Energy & Entropy & $\begin{array}{c}\text { Sum } \\
\text { average }\end{array}$ & $\begin{array}{c}\text { Sum } \\
\text { variance }\end{array}$ & $\begin{array}{c}\text { Sum } \\
\text { entropy }\end{array}$ \\
\hline \multirow{2}{*}{$\begin{array}{c}\text { Back } \\
\text { view }\end{array}$} & 35.2932 & 0.4957 & 0.7436 & 9.6661 & 127.858 & 0.7374 \\
\cline { 2 - 7 } & 36.0650 & 0.7971 & 0.7490 & 9.8416 & 130.627 & 0.7418 \\
\hline \multirow{2}{*}{ Mid view } & 35.5560 & 0.4963 & 0.7441 & 9.7258 & 128.826 & 0.7378 \\
\cline { 2 - 7 } & 36.0565 & 0.4973 & 0.7482 & 9.8408 & 130.626 & 0.7412 \\
\hline \multirow{2}{*}{ Side view } & 35.7504 & 0.4962 & 0.7491 & 9.7762 & 129.488 & 0.7420 \\
\cline { 2 - 7 } & 35.7439 & 0.4962 & 0.7485 & 9.7713 & 129.474 & 0.7415 \\
\hline
\end{tabular}

The proposed image is then tested on the Flair modality based MICCAI brain MRI dataset shown in Table 4. 
From this table, it is observed that, proposed heuristic algorithm and active contour technique is efficient in extracting the tumor section from the Flair modality dataset. In order to confirm the efficiency, the extracted tumor is then compared with the ground truth offered by the expert member. During this comparison work, the image similarity measures, such as Jaccard, Dice, False Positive Rate (FPR) and False Negative Rate (FNR) are recorder as depicted in Table 5. From the average values of Table 5, one can confirm that, proposed approach is very efficient in extracting the tumor from the Flair modality based brain MRI.

Table 4 Test images considered from MICCAI dataset

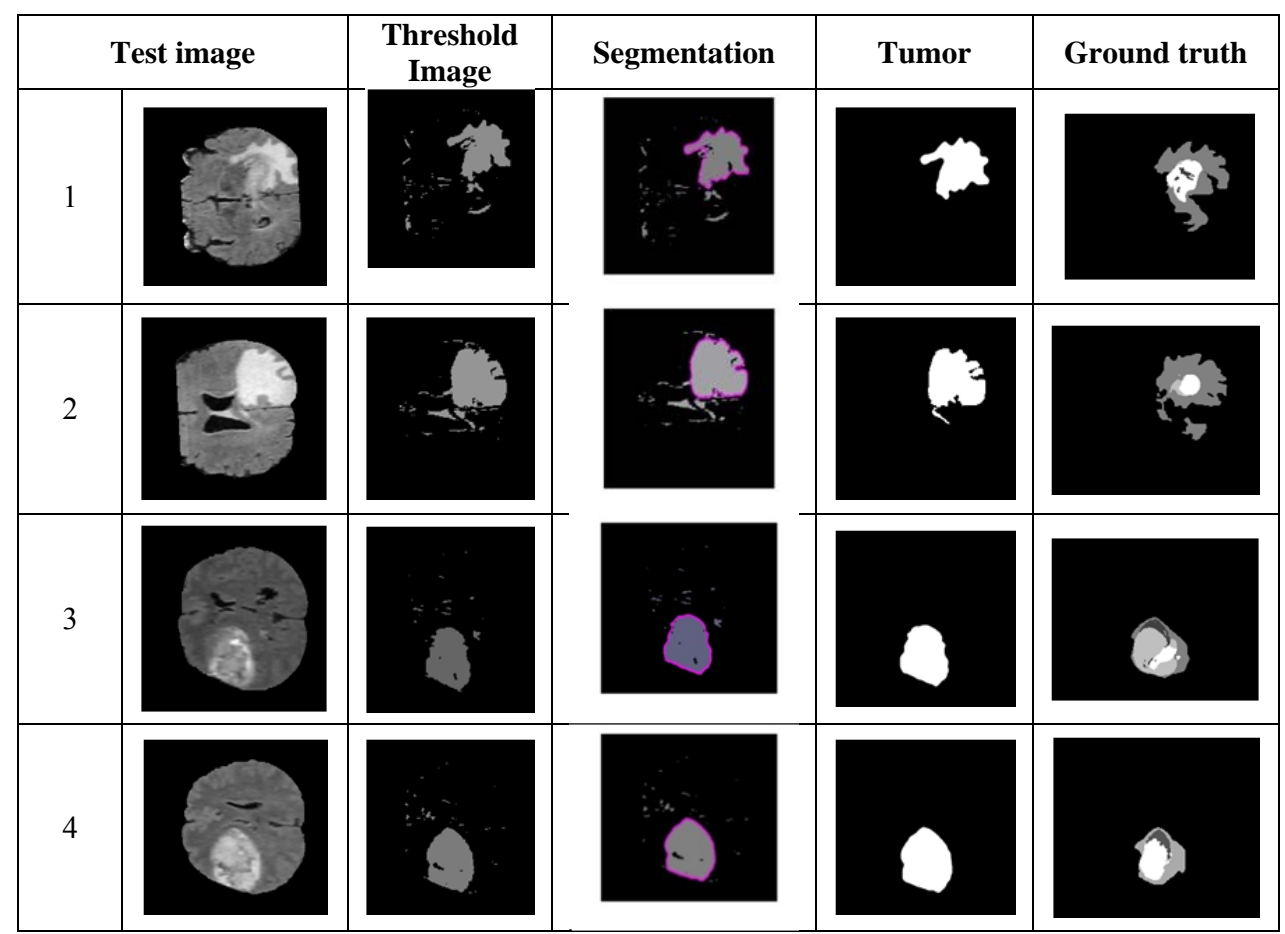

Table 5 Image similarity obtained with the MICCAI dataset

\begin{tabular}{|c|c|c|c|c|}
\hline Image & Jaccard & Dice & FPR & FNR \\
\hline 1 & 0.8155 & 0.8984 & 0.0666 & 0.1302 \\
\hline 2 & 0.8718 & 0.9315 & 0.1112 & 0.0313 \\
\hline 3 & 0.8121 & 0.8963 & 0.1123 & 0.0968 \\
\hline 4 & 0.8616 & 0.9257 & 0.1495 & 0.0096 \\
\hline Average & 0.8402 & 0.9130 & 0.1090 & 0.0670 \\
\hline
\end{tabular}

Finally, the clinical significance of the proposed technique is confirmed against the brain MRI images obtained from the imaging centre. Compared to other datasets, this image is a complex image, since the patient details and the modality are implanted inside the brain image. Hence, the tumor section is enhanced using the bi-level thresholding and the required tumor section is extracted using the Active Contour approach. From Table 6 , it is noted that, the proposed procedure in this paper is efficient in extracting the tumor. In future, this tumor can be validated using the expert member. From this research work, a Matlab based tool is developed to assist the decision making process of brain tumor and the tool is tested against the benchmark image datasets and the real time clinical images. 


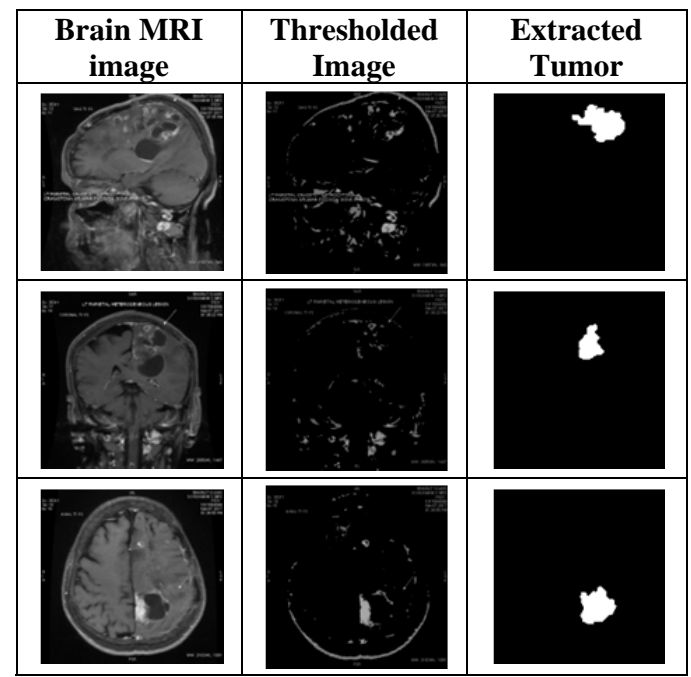

\section{CONCLUSION}

This work proposes an automated tool to mine and analyze the abnormal region of the brain MR image. The work implements the Bat algorithm and Kapur's entropy based procedure to enhance the tumor section and the Active Contour based approach to segment the tumor from thresholded image. The proposed approach is initially tested on well known image datasets existing in the literature. The performance of the proposed technique is confirmed by measuring the image quality measures and image shape measures based the GLCM. The experimental result demonstrates that, proposed approach is very efficient in extracting the tumor section from the chosen test images. The result obtained with the MICCAI dataset confirms that, the proposed approach is efficient in providing better values of the image similarity index, such as the Jaccard, Dice, FPR and FNR.. Finally the clinical significance of the proposed approach is tested by implementing the proposed procedure on real time clinical brain MR images. The results confirmed that, proposed approach offers better result on the clinical images, hence in future, it can be used to analyse the clinical brain MRI to support the decision making and the treatment planning process.

\section{ACKNOWLEDGMENT}

The authors of the paper would like to acknowledge the support provided by Bharat Education and Research Foundation (BERF), academic wing of Bharat Scans Private Limited for supporting the research by providing real clinical images of the brain MRI. Bharat scans is the forefront in the field of medical imaging and other diagnostic services in Chennai, Tamilnadu, India

\section{REFERENCES}

[1] R.Yogamangalam, and B. Karthikeyan, "Segmentation Techniques Comparison in Image Processing ," International Journal of Engineering and Technology (IJET), vol.5, no.1, pp.307-313, 2013.

[2] N. G. Thangamma, and S. Prasanna, "Design And Development Of Medical Image Processing Techniques and to Study their Applications Using Graphical System Design in Ovarian Cancer," International Journal of Engineering and Technology (IJET), vol.8, no.2, pp.1252-1255, 2016.

[3] Arif Widyatama, Okto Dinaryanto, Indarto, and Deendarlianto, "The Application of Digital Image Processing to Study Slug Flow Characteristics in A Horizontal Pipe," International Journal of Engineering and Technology (IJET), vol.8, no.6, pp.2654-2663, 2017.

[4] G.E. Hemamalini, and J Prakash, "Medical Image Analysis of Image Segmentation and Registration Techniques," International Journal of Engineering and Technology (IJET), vol.8, no.5, pp.2234-2241, 2016.

[5] S. Samanta, S. Acharjee, A. Mukherjee , D. Das and N. Dey, "Ant Weight Lifting Algorithm for Image Segmentation," IEEE International Conference on Computational Intelligence and Computing Research (ICCIC), Madurai, Dec 26-28, pp. 1- 5, 2013. DOI: 10.1109/ICCIC.2013.6724160.

[6] S. Bose, A. Mukherjee, Madhulika, S. Chakraborty, S. Samanta, and N. Dey, "Parallel image segmentation using multi-threading and k-means algorithm," IEEE International Conference on Computational Intelligence and Computing Research (ICCIC), Madurai, Dec 26-28, 2013. DOI: 10.1109/ICCIC.2013.6724171.

[7] M. Tuba, "Multilevel image thresholding by nature-inspired algorithms: A short review," Computer Science Journal of Moldova, vol. 22, pp.318-38, 2014.

[8] R. Kumar, F.A. Talukdar, N. Dey, A. S. Ashour, V. Santhi, V. E. Balas, and F. Shi, "Histogram thresholding in image segmentation: a joint level set method and lattice boltzmann method based approach," Advances in Intelligent Systems and Computing, vol.455, pp 529-539, 2016.

[9] P. Roy, S. Goswami, S. Chakraborty, A. Taher Azar, and N. Dey, "Image segmentation using rough set theory: A review," International Journal of Rough Sets and Data Analysis (IJRSDA), vol.1, no.2, pp.62-74, 2014. 
[10] B. Akay, "A study on particle swarm optimization and artificial bee colony algorithms for multilevel thresholding," Applied Soft Computing Journal, vol. 13, no. 6, pp. 3066-3091, 2013.

[11] Palani T Krishnan, Parvathavarthini Balasubramanian, Chitra Krishnan, "Segmentation of brain regions by integrating meta heuristic multilevel threshold with markov random field," Current Medical Imaging Reviews, vol.12, no.1, pp. 4-12, 2016.

[12] X. S. Yang, Nature-Inspired Metaheuristic Algorithms, Luniver Press, Frome, UK, 2nd edition, 2011.

[13] B. Joyce Preethi, R. Angel Sujitha, and V. Rajinikanth, "Otsu based Multi-level Image Segmentation using Brownian Bat Algorithm," International Journal of Computer Applications, ICCCMIT 2014, no.3, pp. 10-16, 2015.

[14] V. Rajinikanth, J.P. Aashiha, and A .Atchaya, "Gray-level histogram based multilevel threshold selection with bat algorithm," International Journal of Computer Applications, vol.93, no.16, pp.1-8, 2014.

[15] Suresh Chandra Satapathy, N. Sri Madhava Raja, V. Rajinikanth, Amira. S.. Ashour, and Nilanjan Dey, "Multi-level image thresholding using Otsu and chaotic bat algorithm," Neural Computing and Applications, pp.1-23, 2016. DOI: 10.1007/s00521-016$2645-5$.

[16] X-S. Yang, "Bat algorithm: Literature review and applications," International Journal of Bio-Inspired Computation, vol. 5, no.3, pp.141-149. 2013.

[17] J. N. Kapur, P. K. Sahoo, and A. K. C. Wong, "A new method for gray-level picture thresholding using the entropy of the histogram," Computer Vision, Graphics, and Image Processing, vol. 29, no. 3, pp. 273-285, 1985.

[18] A.K. Bhandari, A.Kumar, and G.K Singh, "Modified artificial bee colony based computationally efficient multilevel thresholding for satellite image segmentation using Kapur's, Otsu and Tsallis functions," Expert Systems with Applications, vol. 42, pp.1573-1601, 2015.

[19] V. S. Lakshmi, S.G. Tebby, D. Shriranjani, and V. Rajinikanth, "Chaotic cuckoo search and Kapur/Tsallis approach in segmentation of t.cruzi from blood smear images," International Journal of Computer Science and Information Security (IJCSIS), vol.14, CIC 2016, pp. 51-56, 2016.

[20] K.S. Manic, R.K. Priya, and V Rajinikanth, "Image Multithresholding based on Kapur/Tsallis Entropy and Firefly Algorithm," Indian Journal of Science and Technology, vol.9, no.12, 89949, 2016.

[21] N Sri Madhava Raja, R Vishnupriya, Kapur's Entropy and Cuckoo Search Algorithm Assisted Segmentation and Analysis of RGB Images, Indian Journal of Science and Technology, vol.9, no.17, pp. 89936, 2016.

[22] N. Houhou, J-P. Thiran, and X. Bresson, "Fast texture segmentation model based on the shape operator and active contour," IEEE Conference on Computer Vision and Pattern Recognition, CVPR 2008, Anchorage, AK, pp.1 - 8, 2008.

[23] X. Bresson, S. Esedoglu, P. Vandergheynst, J-P. Thiran, and S. Osher, "Fast global minimization of the active contour/snake model," J. Math. Imaging Vis., Vol.28, no. 2, pp.151-167, 2007.

[24] N. S.M. Raja, V. Rajinikanth, and K. Latha, "Otsu Based Optimal Multilevel Image Thresholding Using Firefly Algorithm," Modelling and Simulation in Engineering, vol. 2014, Article ID 794574, 17 pages, 2014.

[25] B. Abhinaya, and N.S.M. Raja, "Solving multi-level image thresholding problem-An analysis with cuckoo search algorithm," Advances in Intelligent Systems and Computing, vol. 339, pp. 177-186, 2015.

[26] V. Rajinikanth, and M.S. Couceiro, "RGB histogram based color image segmentation using firefly algorithm," Procedia Computer Science, vol.46, pp.1449-1457, 2015.

[27] V. Rajinikanth, N.S.M. Raja, and S.C. Satapathy, "Robust color image multi-thresholding using between-class variance and cuckoo search algorithm," in Information Systems Design and Intelligent Applications, Advances in Intelligent Systems and Computing, vol.433, pp. 379-386, 2016.

[28] A. Chaddad, and C. Tanougast, "Quantitative evaluation of robust skull stripping and tumor detection applied to axial MR images," Brain Informatics, vol.3, no.1, pp.53-61, 2016.

[29] V. Rajinikanth, N.S.M. Raja, and K. Latha, "Optimal multilevel image thresholding: an analysis with PSO and BFO algorithms. Aust. J. Basic Appl. Sci., vol. 8, pp. 443-454, 2014.

[30] S. Samanta, N. Dey, P. Das, S.Acharjee, and S.S. Chaudhuri, "Multilevel threshold based gray scale image segmentation using cuckoo search," International Conference on Emerging Trends in Electrical, Communication And Information Technologies -ICECIT, Dec 1223,2012, arXiv:1307.0277.

[31] N. S. Balan, A. S.Kumar, N.S.M. Raja, and V. Rajinikanth, "Optimal Multilevel Image Thresholding to Improve the Visibility of Plasmodium sp. in Blood Smear Images," Advances in Intelligent Systems and Computing, vol.397, pp. 563-571, 2016.

[32] K. Keerthana, T.J. Jayasuriya, N. Sri Madhava Raja, V. Rajinikanth, Retinal Vessel Extraction based on Firefly Algorithm guided Multi-scale Matched Filter, International Journal of Modern Science and Technology, vol.2, no.2, pp. 74-80, 2017.

[33] K. Manickavasagam, S. Sutha and K, Kamalanand, An automated system based on $2 \mathrm{~d}$ empirical mode decomposition and k-means clustering for classification of Plasmodium species in thin blood smear images, BMC Infectious Diseases, vol.14, no.3, pp.1, 2014.

[34] K. Manickavasagam, S. Sutha and K. Kamalanand, Development of systems for classification of different plasmodium species in thin blood smear microscopic images, Journal of Advanced Microscopy Research, vol.9, no.2, pp. 86-92, 2014.

[35] G. K.Vaishnavi, K. Jeevananthan, S. R. Begum, and K. Kamalanand, "Geometrical Analysis of Schistosome Egg Images Using Distance Regularized Level Set Method for Automated Species Identification," Journal of Bioinformatics and Intelligent Control, vol.3, no.2, pp. 147-152, 2014.

[36] Brain Tumor Database (CEREBRIX and BRAINIX), http://www.osirix-viewer.com/datasets/

[37] Brain Tumor Database (BraTS-MICCAI), http://hal.inria.fr/hal-00935640.

[38] http://www.bharatscans.com/

\section{AUTHOR PROFILE}

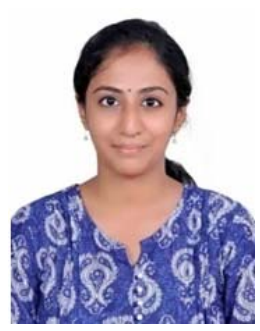

S. Madhuvanthi is currently pursuing B.E. in Electronics and Instrumentation Engineering, St. Joseph's College of Engineering, Chennai 600 119, Tamilnadu, India.

She is currently working in the field of classical and heuristic algorithm assisted medical image segmentation and analysis. She is also interested in developing a low cost tool to assist the clinics related to brain abnormality analysis. 